\title{
The importance of biopsy in clinically diagnosed metastatic lesions in patients with breast cancer
}

\author{
Qing Qu ${ }^{1}$, Yu Zong ${ }^{2}$, Xiao-chun Fei ${ }^{3}$, Xiao-song Chen², Cheng Xu' ${ }^{1}$ Gu-yin Lou ${ }^{1}$ and Kun-wei Shen ${ }^{2 *}$
}

\begin{abstract}
Background: Receptor status discordance, such as estrogen receptor (ER), progesterone receptor (PR) and human epidermal growth factor receptor 2 (HER2) status between primary breast cancer and metastatic lesions has been reported. The aim of this study was to evaluate the biopsy of clinically diagnosed metastatic lesions and to determine the changes in hormonal receptor and HER2 status of the metastatic lesions.
\end{abstract}

Methods: Sixty-three patients with clinically diagnosed metastatic breast cancer underwent an excisional biopsy or core needle aspiration guided by computed tomography/ultrasound. ER, PR and HER2 were assessed by immunohistochemistry (IHC).

Results: A total of 48 metastases (76.2\%) and nine second primary malignancies (14.3\%, seven primary lung cancers and two primary pancreatic cancers) were found. The discrepancies between ER, PR and HER2 status between the primary breast cancer and metastatic lesions were 14.6\%, 16.7\% and 8.3\%, respectively. Six lesions (9.5\%) were proved benign upon biopsy.

Conclusions: The biopsy of clinically suspicious metastatic lesions could histologically confirm the diagnosis of metastasis, evaluate discrepancies between ER, PR and HER2 status and exclude secondary malignancy, which might change the therapeutic strategy for breast cancer patients.

Keywords: Breast cancer, Metastasis, Second malignancy, Biopsy, Receptor status

\section{Background}

Breast cancer is the most common malignancy affecting women in developed countries. In several cities in China, the incidence has increased dramatically over the past 30 years [1]. Breast cancer patients with early stage disease can be cured, although more than $20 \%$ of these patients will eventually develop incurable metastatic disease $[2,3]$. However, the rates of disease-free survival and the overall survival have increased over the years, largely because adjuvant therapy (chemotherapy, radiation therapy or hormone therapy), has helped prevent local and distant failures. The estrogen receptor (ER), progesterone receptor (PR), and human epidermal growth factor receptor 2 (HER2) are important indicators to determine the prognosis of patients with breast cancer. Breast cancers can be classified into five subtypes

\footnotetext{
*Correspondence: kwshenrj@163.com

${ }^{2}$ Comprehensive Breast Health Center, Ruijin Hospital, Shanghai Jiao Tong University School of Medicine, 197, Ruijin 2 Road, Shanghai 200025, People's Republic of China

Full list of author information is available at the end of the article
}

according to the ER, PR, HER2 and other markers: luminal A, luminal B, normal breast-like, basal-like, and HER2 overexpressing tumors. ER, PR and HER2 are essential in determining the use of hormone therapy, chemotherapy and targeted therapy [4-7].

Metastasis is the most frequent reason for treatment failure in breast cancer. Diagnosis of metastasis or relapse usually depends on clinical, biological and radiologic evidence [8]. In the metastatic setting, the characteristics of the primary tumors, such as ER, PR and HER2 status, are important to determine the choice of therapeutic strategy. Oncologists often use primary tumor biomarkers to choose endocrine therapy, chemotherapy or targeted therapy for metastatic disease. However, in the last few years, several studies have demonstrated a significant discordance between hormone receptor (HR) status and HER2 status between primary breast cancer and paired asynchronous metastasis [9-13]. If these therapy-predictive markers change throughout tumor progression, then investigating metastatic lesions would provide additional 
important information about the metastasis, which would enable better management of patients with advanced disease. Due to new therapies, such as targeted therapy and new endocrine therapies, selection of patients using this additional information is crucial for increasing clinical benefit and avoiding unnecessary treatment and toxicities.

The aim of this retrospective study was to investigate the changes in ER, PR and HER2 status in metastatic lesions compared with paired primary breast cancer, and to find changes in the treatment strategies after biopsy confirmation of recurrence with assessment of predictive markers in the patients with suspected breast cancer metastasis.

\section{Patients and methods \\ Patients}

A total of 63 patients were diagnosed and treated in the Comprehensive Breast Health Center of Ruijin Hospital, Shanghai Jiaotong University School of Medicine, Shanghai, China between September 2009 and December 2012. Patients who met the following criteria were considered for further analysis: (1) primary breast cancer totally resected, (2) metachronous lesions with suspected metastasis detected by physical examination, ultrasound or computed tomography (CT) without evidence of primary tumor recurrence, (3) multidisciplinary decision for biopsy by surgeons, medical oncologists, radiation therapists, radiologists and pathologists, and (4) complete clinical and follow-up data. Ultimately, 63 patients met the inclusion criteria and were selected for this study. The metastatic histological assessment could be made at metastatic lesion presentation or later after the completion of several lines of treatment. Patients who only underwent cytological investigation of metastasis were excluded from the study.

\section{Methods}

Tissue specimens were obtained by excisional biopsy or core needle aspiration biopsy under guidance of ultrasound or CT. A representative section of the tumor specimen from each case was selected and stained immunohistochemically with a panel of antibodies including ER, PR and HER2. Routine H\&E-stained sections were reviewed for the histological tumor type. Immunohistochemistry (IHC) staining was performed on formalin-fixed, paraffin-embedded tissues to evaluate ER, PR and HER2 status. The anti-ER antibody and antiPR antibody were both from DAKO (Carpinteria, CA, USA). Positive staining for ER/PR was defined as nuclear staining in more than $1 \%$ of tumor cells. The anti-HER2 antibody was from Roche (Basel, Switzerland). HER2 was evaluated by an experienced pathologist and scored as $0,1+, 2+$, and $3+$ according to the American Society of Clinical Oncology/College of American Pathologists
(ASCO/CAP) guidelines. HER2 negativity was considered as HER2 0 or $1+$, whereas cases with $2+$, or $3+$ would be tested using fluorescence in situ hybridization (FISH). An amplification ratio HER $2 / \mathrm{C}-17$ of $>2$ on FISH test was considered as HER2 positivity.

\section{Results}

\section{Clinical characteristics of patients}

Table 1 summarizes the clinical characteristics of the biopsied patients. The median age at breast cancer

Table 1 Characteristics of biopsied patients

\begin{tabular}{|c|c|}
\hline Characteristics & $n=63(\%)$ \\
\hline Age (years) & 53.1 (30 to 73$)$ \\
\hline RFS (months) & 34.8 (4 to 190$)$ \\
\hline \multicolumn{2}{|l|}{ Tumor size } \\
\hline $\mathrm{T} 1$ & $32(50.8 \%)$ \\
\hline $\mathrm{T} 2$ & $27(42.9 \%)$ \\
\hline T3 & $4(6.3 \%)$ \\
\hline \multicolumn{2}{|l|}{ Lymph node staging } \\
\hline 0 & $24(38.1 \%)$ \\
\hline 1 & $24(38.1 \%)$ \\
\hline 2 & $9(14.3 \%)$ \\
\hline 3 & $6(9.5 \%)$ \\
\hline \multicolumn{2}{|l|}{ Histologic type } \\
\hline IDC & $62(98.4 \%)$ \\
\hline Apocrine carcinoma & $1(1.6 \%)$ \\
\hline \multicolumn{2}{|l|}{ Molecule subtype } \\
\hline Luminal & $32(50.8 \%)$ \\
\hline TNBC & $24(38.1 \%)$ \\
\hline HER2 positive & $7(11.1 \%)$ \\
\hline \multicolumn{2}{|l|}{ Adjuvant chemotherapy } \\
\hline Yes & $47(74.6 \%)$ \\
\hline No & $16(25.4 \%)$ \\
\hline \multicolumn{2}{|c|}{ Adjuvant endocrine therapy } \\
\hline Yes & $32(50.8 \%)$ \\
\hline No & $31(49.2 \%)$ \\
\hline \multicolumn{2}{|l|}{ Adjuvant radiotherapy } \\
\hline Yes & $29(46.0 \%)$ \\
\hline No & $34(54.0 \%)$ \\
\hline \multicolumn{2}{|c|}{ Location of suspicious lesions } \\
\hline Viscera $^{a}$ & $48(76.2 \%)$ \\
\hline Soft tissues ${ }^{b}$ & $15(23.8 \%)$ \\
\hline \multicolumn{2}{|c|}{ Number of suspicious lesions } \\
\hline Single & $11(17.5 \%)$ \\
\hline Multiple & $52(82.5 \%)$ \\
\hline
\end{tabular}

Abbreviations: IDC, invasive ductal carcinoma, RFS, relapse free survival, TNBC, triple negative breast cancer, ${ }^{a}$ Viscera: liver, pleura, lung, and ovary; ${ }^{\text {b}}$ Soft tissues: lymph node and soft tissue. 
diagnosis was 53.1 years (range, 30 to 73 years); most patients had invasive ductal histology (98.4\%), and most primary tumors were the luminal subtype.

Table 2 summarizes the biopsy characteristics in these patients. Most patients had undergone biopsy of the lung (38.1\%), and the most frequent guidance was using CT (61.9\%).

\section{Receptor expression discordance between primary breast} cancer and metastatic lesions

Overall, 48 metastases $(76.2 \%)$ were confirmed through biopsy. ER and PR status changed between the primary breast tumor and metastatic lesions in $14.6 \%$ and $16.7 \%$ of patients, respectively (Table 3 ). There were only four (8.3\%) cases that showed a discrepancy in HER2 status (Figure 1). According to the HR and HER2 status of the metastatic lesions, the choice of treatment for the patients was determined at a multidisciplinary treatment meeting. The treatment for the patients without any discrepancy between the primary tumor and metastatic lesions was chosen according to the characteristics of the primary tumors. For the patients with a discrepancy between HR and HER2 status, fourteen patients received modified therapeutic strategies, including ten hormone therapies and four targeted therapies due to a switch in receptors status.

\section{Second primary malignancy}

In our study, a second primary malignancy was defined as one with a different histological type or different components external to the breast. Nine second primary malignancies (14.3\%) were diagnosed upon biopsy, including seven primary lung cancers (Figure 2) and two primary pancreatic cancers.

Table 2 Characteristics of rebiopsies

\begin{tabular}{ll}
\hline Characteristics & $\mathbf{n = 6 3 ( \% )}$ \\
\hline Site of rebiopsy & $24(38.1 \%)$ \\
Lung & $12(19.0 \%)$ \\
Chest wall & $9(14.3 \%)$ \\
Liver & $12(19.0 \%)$ \\
Lymph nodes & $2(3.2 \%)$ \\
Pancreas & $1(1.6 \%)$ \\
Abdominal wall & $1(1.6 \%)$ \\
Pelvis & $1(1.6 \%)$ \\
Mediastinum & $1(1.6 \%)$ \\
Bone & \\
Guide for rebiopsy & $39(61.9 \%)$ \\
CT & $16(25.4 \%)$ \\
Ultrasound & $8(12.7 \%)$ \\
Incision &
\end{tabular}

Table 3 Change in estrogen receptor and progesterone receptor (ER/PR) and human epidermal growth factor receptor 2 (HER2) status in primary and metastatic tumors

\begin{tabular}{lll}
\hline Primary tumor & Metastatic lesions & Case number \\
\hline$E R-$ & $E R+$ & 2 \\
$E R+$ & $E R-$ & 5 \\
$P R-$ & $P R+$ & 1 \\
$P R+$ & $P R-$ & 7 \\
HER2 - & HER2 + \\
HER2 + & HER2 - & 2 \\
\hline
\end{tabular}

\section{Benign lesion}

Six patients (9.5\%) did not have any evidence of relapse or metastasis upon biopsy. These patients were followed every three months and to date have shown no disease progression.

\section{Discussion}

Breast cancer threatens womens' health all over the world. Breast cancer is now the second most common malignancy in China [14]. Nearly one half of these patients will eventually develop metastases. In the metastatic setting, the choice of systemic treatment is often based on the biomarker characteristics of the primary tumor, including ER, PR and HER2 status. The breast cancer subtypes defined by ER, PR, and HER2 are helpful to direct treatment and choose endocrine therapy, molecular-targeted therapy and cytotoxic chemotherapy.

Firstly, our study shows that ER, PR, and HER2 are lost and gained in a considerable proportion of patients throughout tumor progression: loss of ER in five cases, gain of ER in two cases, loss of PR in seven cases, gain of PR in one case, loss of HER2 in two cases, and gain of HER2 in two cases. Several studies have also demonstrated the discordance between the primary tumor and metastatic lesions in recent decades $[10,13,15,16]$. Published studies have found discordance rates for ER status ranging from 10.2 to $56 \%$, PR status ranging from 24.8 to $48.6 \%$, and HER2 status ranging from 2.9 to $16 \%$ [15-23]. Jenson's study has shown that ER status of the metastases changed in 12 to $13 \%$ of cases, and HER2 status changed in 5 to $8 \%$ [17]. ER/PR and HER2 statuses may be modified by treatment or during disease progression. Liu et al. reported that in asynchronous liver metastases, the change in ER, PR and HER2 was $30.4 \%, 54.3 \%$ and $10.9 \%$, respectively. However, in synchronous liver metastases, the change in ER, PR and HER2 were $0,33.3 \%$ and $8.3 \%$ respectively [18]. Lindstrom and colleagues demonstrated that one in three patients with breast cancer experience alteration of $\mathrm{HR}$ status, and $15 \%$ of patients experience a change in HER2 status during tumor progression. In addition, they found 

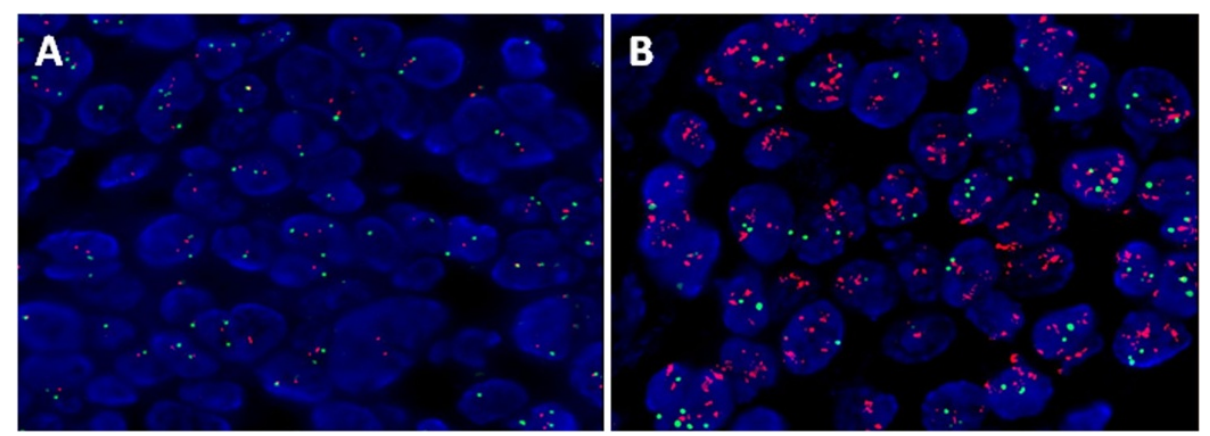

Figure 1 Microscopic findings of HER2 in (A) primary breast tumor (FISH-, magnification x 1,250); (B) liver metastasis (FISH+, magnification $\times 1,250$ ).

that patients with ER-positive primary tumors that changed to ER-negative metastases had an increased mortality risk compared with patients with stable ER-positive tumors. The understanding of tumor dissemination by clarifying the instability of clinically used tumor markers throughout tumor progression could be used to infer both biologic and therapeutic implications in the metastatic setting [19]. The discordance of ER and HER2 in the relapse setting would introduce additional therapeutic choices. The clinical implication of this discordance is important, the loss of HR and HER2 generally indicates resistance to endocrine therapy and trastuzumab, respectively and therefore these patients would benefit from a change of treatment strategy. A total of
14 patients required modification of their treatment strategies in our study, including hormone therapy and targeted therapy because of the altered characteristics in metastatic lesions. Seven patients received endocrine therapy because of the switch of hormone receptor from negative to positive; three patients received chemotherapy because of the negative hormone receptor status in the metastatic lesions which most likely indicates a resistance to endocrine therapy. Two patients were eligible for treatment with trastuzumab because of HER2 status switch to positive; two patients did not use trastuzumab in case of negativity of HER2 in metastatic lesions which probably means a resistance to trastuzumab. Since tumor instability is seen throughout tumor progression,

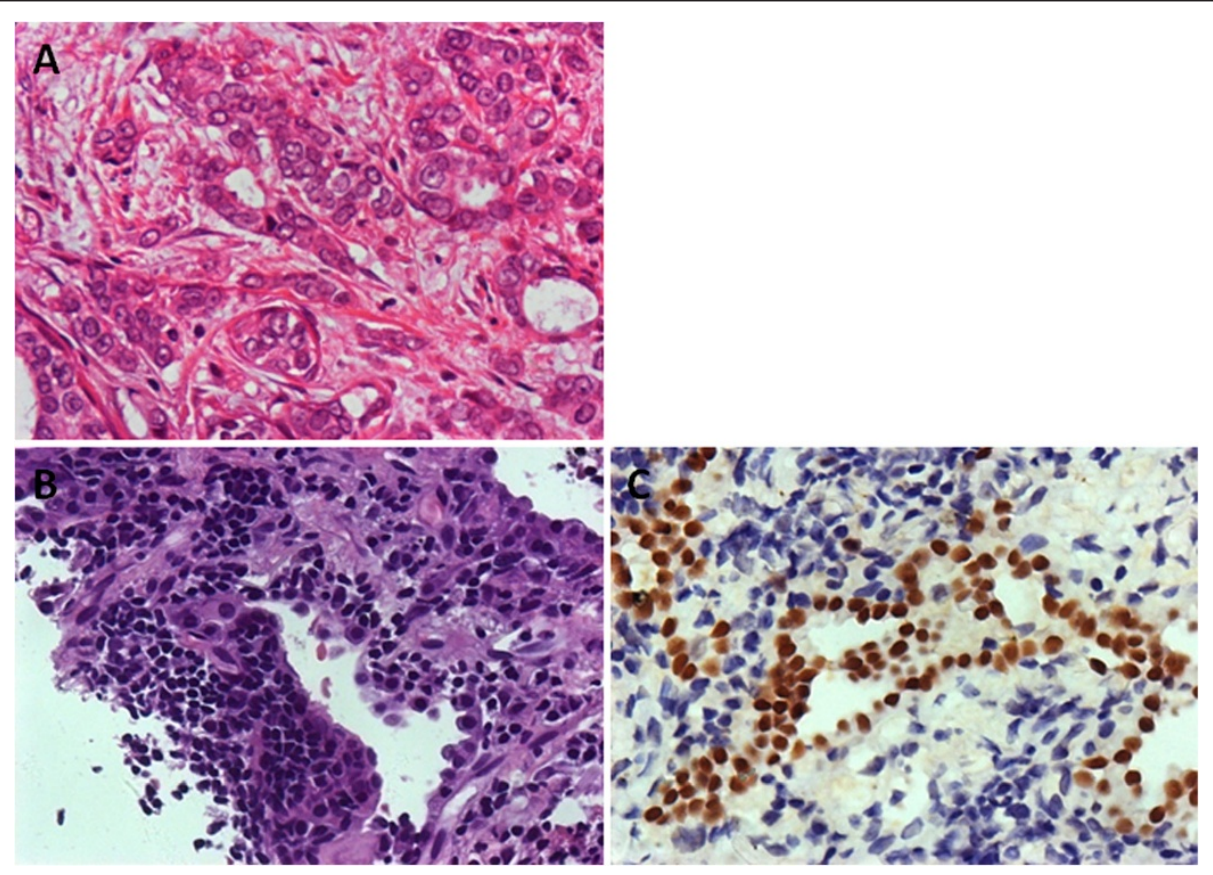

Figure 2 Microscopic findings of (A) primary tumor (magnification x 200), (B) site of lung lesion (magnification $\times 200$ ); and (C) site of primary lung cancer (IHC TTF-1+, magnification x 200). 
this dynamic highlights the potential need for taking biopsies in a consecutive manner in the advanced setting to optimize clinical decision-making for the patient.

There are several explanations for these alterations. The characteristics of the metastatic lesions could be influenced by factors such as clonal selection by microenvironment and clonal changes induced by adjuvant therapies [24-26]. Additionally, certain factors can influence the IHC results. In the process of IHC staining, many factors could affect results such as fixation time, staining methodology and the size of tissue blocks [27]. Therefore, according to the European Society for Medical Oncology (ESMO) guideline, a biopsy should be performed if there is one single lesion, as well as when the patient has a history of more than one cancer, and when there is suspicion of an alternative diagnosis [28].

Secondly, we found nine second primary malignancies external to the breast through the biopsy of clinically diagnosed metastatic lesions. A number of studies have reported that patients with breast cancer have a high risk of developing a second malignancy, with standardized incidence ratios (SIRs) ranging from 1.15 to 1.6 [29-32]. Brown et al. calculated that the SIR for second cancers was 1.15 in patients with a history of breast cancer [29]. Kirova et al. reported that the SIR of the primary lung cancer in French patients with a history of the breast cancer is 1.2, but this finding was not significant [30]. Our observation of an increased risk of second primary lung cancer confirms previous findings. Rena et al. reported that for a single lung lesion in patients with breast cancer, the rate of primary lung cancer and lung metastasis was $48.1 \%$ and $34.2 \%$, respectively [33]. Jensen et al. also showed that biopsies of the suspicious metastatic lesions were benign disease or other malignancies in $14 \%$ of the patients [17]. In our research, all seven patients with primary lung cancers, confirmed by core needle biopsy, finally had surgery or chemotherapy for the lung cancer. The mechanism involved in the development of second malignancy has not been fully identified. It may be related to the treatment of the breast cancer, such as radiation therapy and anti-estrogen therapy [29,30,34-36].

Thirdly, in our study six patients had a confirmed benign lesion through rebiopsy. The pathologists did not find any evidence of metastasis. These six patients have received regular follow-up every three months. In their study, Rena et al. also reported the rate of the benign lesion of single lung lesion after breast surgery was $17.7 \%$ [33].

There are several limitations inherent in this study. This analysis was based on a single-center, retrospective study, and therefore we are planning to design prospective studies in the future. In addition, the database spans a short time period (2009 to 2012), during which there has not been enough follow-up data to determine the relationship between the prognosis and the discrepancies of ER, PR and HER2 status.

\section{Conclusions}

In summary, biopsy of suspicious lesions can confirm the relapse of the tumor, exclude the second primary tumor, identify the status of ER, PR and HER2, and allow some patients with breast cancer to benefit from hormone therapy and/or anti-HER2 treatment. The discordance in ER and PR receptor expression between the primary breast tumor and the corresponding metastatic lesions is high, whereas HER2 status remains relatively constant. Therapeutic strategies could be changed after the biopsy because of changes in the tumor characteristics and, therefore, the patients could receive the most effective treatment and avoid unnecessary toxicity. We recommend that biopsy of suspicious metastases and reassessment of ER, PR and HER2 status should become a routine procedure in the treatment of breast cancer patients.

\section{Abbreviations}

$C T$ : computed tomography; ER: estrogen receptor; ESMO: European Society for Medical Oncology; FISH: fluorescence in situ hybridization;

H\&E: hematoxylin and eosin; HR: hormone receptor; HER2: human epidermal growth factor receptor 2; IHC: immunohistochemistry; FISH: Fluorescence in situ hybridization PR, Progesterone receptor; SIRs: standardized incidence ratios.

\section{Competing interests}

The authors have no financial conflicts of interest to declare.

\section{Authors' contributions}

$\mathrm{QQ}, \mathrm{X}-\mathrm{SC}$ and CX participated in the design of the study and drafted the manuscript. QQ, CX and G-YL participated in data acquisition and analysis. X-CF carried out the pathological immunoassays. QQ, YZ and K-WS carried out the literature review and revised the manuscript. All authors read and approved the final manuscript.

\section{Acknowledgement}

This research is supported by the Scientific Research Project of Shanghai Municipal Health Bureau (20134Y039).

\section{Author details}

'Department of Oncology, Ruijin Hospital, Shanghai Jiao Tong University School of Medicine, Shanghai 200025, China. ${ }^{2}$ Comprehensive Breast Health Center, Ruijin Hospital, Shanghai Jiao Tong University School of Medicine, 197, Ruijin 2 Road, Shanghai 200025, People's Republic of China.

${ }^{3}$ Department of Pathology, Ruijin Hospital, Shanghai Jiao Tong University School of Medicine, Shanghai 200025, China.

Received: 3 October 2013 Accepted: 30 March 2014

Published: 10 April 2014

\section{References}

1. Linos E, Spanos D, Rosner BA, Linos K, Hesketh T, Qu JD, Gao YT, Zheng W, Colditz GA: Effects of reproductive and demographic changes on breast cancer incidence in China: a modeling analysis. J Natl Cancer Inst 2008, 100:1352-1360.

2. Berry DA, Cronin KA, Plevritis SK, Fryback DG, Clarke L, Zelen M, Mandelblatt JS, Yakovlev AY, Habbema JD, Feuer EJ: Effect of screening and adjuvant therapy on mortality from breast cancer. N Engl J Med 2005, 353:1784-1792.

3. Effects of chemotherapy and hormonal therapy for early breast cancer on recurrence and 15-year survival: an overview of the randomised trials. Lancet 2005, 365:1687-1717. 
4. Perou CM, Sorlie T, Eisen MB, van de Rijn M, Jeffrey SS, Rees CA, Pollack JR, Ross DT, Johnsen $H$, Akslen LA, Fluge O, Pergamenschikov A, Williams C, Zhu SX, Lønning PE, Børresen-Dale AL, Brown PO, Botstein D: Molecular portraits of human breast tumours. Nature 2000, 406:747-752.

5. Sorlie T, Perou CM, Tibshirani R, Aas T, Geisler S, Johnsen H, Hastie T, Eisen MB, van de Rijn M, Jeffrey SS, Thorsen T, Quist H, Matese JC, Brown PO, Botstein D, Lønning PE, Børresen-Dale AL: Gene expression patterns of breast carcinomas distinguish tumor subclasses with clinical implications. Proc Natl Acad Sci U S A 2001, 98:10869-10874.

6. Sorlie T, Tibshirani R, Parker J, Hastie T, Marron JS, Nobel A, Deng S, Johnsen H, Pesich R, Geisler S, Demeter J, Perou CM, Lønning PE, Brown PO, Børresen-Dale AL, Botstein D: Repeated observation of breast tumor subtypes in independent gene expression data sets. Proc Natl Acad Sci U S A 2003, 100:8418-8423.

7. McDermott SP, Wicha MS: Targeting breast cancer stem cells. Mol Oncol 2010, 4:404-419.

8. Amir E, Clemons M: Should a biopsy be recommended to confirm metastatic disease in women with breast cancer? Lancet Oncol 2009, 10:933-935.

9. Gutierrez MC, Detre S, Johnston S, Mohsin SK, Shou J, Allred DC, Schiff R, Osborne CK, Dowsett M: Molecular changes in tamoxifen-resistant breast cancer: relationship between estrogen receptor, HER2, and p38 mitogen-activated protein kinase. J Clin Oncol 2005, 23:2469-2476.

10. Johnston SR, Saccani-Jotti G, Smith IE, Salter J, Newby J, Coppen M, Ebbs $S R$, Dowsett M: Changes in estrogen receptor, progesterone receptor, and pS2 expression in tamoxifen-resistant human breast cancer. Cancer Res 1995, 55:3331-3338.

11. Simmons C, Miller N, Geddie W, Gianfelice D, Oldfield M, Dranitsaris G, Clemons MJ: Does confirmatory tumor biopsy alter the management of breast cancer patients with distant metastases? Ann Oncol 2009, 20:1499-1504

12. Kuukasjarvi T, Kononen J, Helin H, Holli K, Isola J: Loss of estrogen receptor in recurrent breast cancer is associated with poor response to endocrine therapy. J Clin Oncol 1996, 14:2584-2589.

13. Gancberg D, Di Leo A, Cardoso F, Rouas G, Pedrocchi M, Paesmans M, Verhest A, Bernard-Marty C, Piccart MJ, Larsimont D: Comparison of HER2 status between primary breast cancer and corresponding distant metastatic sites. Ann Oncol 2002, 13:1036-1043.

14. Hao XS, Chen KS, Liu H: Analysis of female breast cancer descriptive epidemiology in Tianjin, China. Asian Pac J Cancer Prev 2002, 3:143-148.

15. Broom RJ, Tang PA, Simmons C, Bordeleau L, Mulligan AM, O'Malley FP, Miller N, Andrulis IL, Brenner DM, Clemons MJ: Changes in estrogen receptor, progesterone receptor and HER2/neu status with time: discordance rates between primary and metastatic breast cancer. Anticancer Res 2009, 29:1557-1562.

16. Zidan J, Dashkovsky I, Stayerman C, Basher W, Cozacov C, Hadary A: Comparison of HER2 overexpression in primary breast cancer and metastatic sites and its effect on biological targeting therapy of metastatic disease. Br J Cancer 2005, 93:552-556.

17. Jensen JD, Knoop A, Ewertz M, Laenkholm AV: ER, HER2, and TOP2A expression in primary tumor, synchronous axillary nodes, and asynchronous metastases in breast cancer. Breast Cancer Res Treat 2012, 132:511-521.

18. Liu J, Deng H, Jia W, Zeng Y, Rao N, Li S, Jin L, Wu J, Song E, Su F: Comparison of ER/PR and HER2 statuses in primary and paired liver metastatic sites of breast carcinoma in patients with or without treatment. J Cancer Res Clin Oncol 2012, 138:837-842.

19. Lindstrom LS, Karlsson E, Wilking UM, Johansson U, Hartman J, Lidbrink EK, Hatschek T, Skoog L, Bergh J: Clinically used breast cancer markers such as estrogen receptor, progesterone receptor, and human epidermal growth factor receptor 2 are unstable throughout tumor progression. J Clin Oncol 2012, 30:2601-2608.

20. Hoefnagel LD, Moelans CB, Meijer SL, van Slooten HJ, Wesseling P, Wesseling J, Westenend PJ, Bart J, Seldenrijk CA, Nagtegaal ID, Oudejans J, van der Valk $\mathrm{P}$, van Gils $\mathrm{CH}$, van der Wall E, van Diest PJ: Prognostic value of estrogen receptor alpha and progesterone receptor conversion in distant breast cancer metastases. Cancer 2012, 118:4929-4935.

21. Gong Y, Booser DJ, Sneige N: Comparison of HER2 status determined by fluorescence in situ hybridization in primary and metastatic breast carcinoma. Cancer 2005, 103:1763-1769.
22. Liedtke C, Broglio K, Moulder S, Hsu L, Kau SW, Symmans WF, Albarracin C, Meric-Bernstam F, Woodward W, Theriault RL, Kiesel L, Hortobagyi GN, Pusztai L, Gonzalez-Angulo AM: Prognostic impact of discordance between triple-receptor measurements in primary and recurrent breast cancer. Ann Oncol 2009, 20:1953-1958.

23. Thompson AM, Jordan LB, Quinlan P, Anderson E, Skene A, Dewar JA, Purdie CA: Prospective comparison of switches in biomarker status between primary and recurrent breast cancer: the Breast Recurrence In Tissues Study (BRITS). Breast Cancer Res 2010, 12:R92.

24. Fidler IJ, Kripke ML: Metastasis results from preexisting variant cells within a malignant tumor. Science 1977, 197:893-895.

25. Chambers AF, Groom AC, MacDonald IC: Dissemination and growth of cancer cells in metastatic sites. Nat Rev Cancer 2002, 2:563-572.

26. Hawkins RA, Tesdale AL, Anderson ED, Levack PA, Chetty U, Forrest AP: Does the oestrogen receptor concentration of a breast cancer change during systemic therapy? Br J Cancer 1990, 61:877-880.

27. Pusztai L, Viale G, Kelly CM, Hudis CA: Estrogen and HER2 receptor discordance between primary breast cancer and metastasis. Oncologist 2010, 15:1164-1168.

28. Foukakis T, Astrom G, Lindstrom L, Hatschek T, Bergh J: When to order a biopsy to characterise a metastatic relapse in breast cancer. Ann Oncol 2012, 23(Suppl 10):x349-x353.

29. Brown LM, Chen BE, Pfeiffer RM, Schairer C, Hall P, Storm H, Pukkala E, Langmark F, Kaijser M, Andersson M, Joensuu H, Fosså SD, Travis LB: Risk of second non-hematological malignancies among 376,825 breast cancer survivors. Breast Cancer Res Treat 2007, 106:439-451.

30. Kirova YM, De Rycke Y, Gambotti L, Pierga JY, Asselain B, Fourquet A: Second malignancies after breast cancer: the impact of different treatment modalities. Br J Cancer 2008, 98:870-874.

31. Shilkrut M, Belkacemi $Y$, Kuten A: Secondary malignancies in survivors of breast cancer: how to overcome the risk. Crit Rev Oncol Hematol 2012, 84(Suppl 1):e86-e89.

32. Vidal-Millan S, Zeichner-Gancz I, Flores-Estrada D, Vela-Rodriguez BE, Vazquez-Lopez MI, Robles-Vidal CD, Ramirez-Ugalde MT, Chavez-MacGregor M: A descriptive study of second primary malignancies associated to breast cancer in a Mexican Hispanic population. Med Oncol 2005, 22:17-22.

33. Rena O, Papalia E, Ruffini E, Filosso PL, Oliaro A, Maggi G, Casadio C: The role of surgery in the management of solitary pulmonary nodule in breast cancer patients. Eur J Surg Oncol 2007, 33:546-550.

34. Lee KD, Chen SC, Chan CH, Lu CH, Chen CC, Lin JT, Chen MF, Huang SH, Yeh CM, Chen MC: Increased risk for second primary malignancies in women with breast cancer diagnosed at young age: a population-based study in Taiwan. Cancer Epidemiol Biomarkers Prev 2008, 17:2647-2655.

35. Trentham-Dietz A, Newcomb PA, Nichols HB, Hampton JM: Breast cancer risk factors and second primary malignancies among women with breast cancer. Breast Cancer Res Treat 2007, 105:195-207.

36. Prochazka M, Hall P, Granath F, Czene K: Family history of breast cancer and young age at diagnosis of breast cancer increase risk of second primary malignancies in women: a population-based cohort study. Br J Cancer 2006, 95:1291-1295.

\section{doi:10.1186/1477-7819-12-93}

Cite this article as: Qu et al.: The importance of biopsy in clinically diagnosed metastatic lesions in patients with breast cancer. World Journal of Surgical Oncology 2014 12:93. 